\title{
Editorial
}

\section{Obstetric anaesthesia in patients with primary pulmonary hypertension}

In this issue Breen and Janzen report successful anaesthetic management of Caesarean delivery in a young patient with primary pulmonary hypertension (PPH). A comprehensive review of the pathophysioloy and management of PPH has recently been published by Rich. ${ }^{1}$ This very rare condition may occasionally present for the first time during pregnancy, particularly during the second and third trimester, when cardiovascular demands become important. ${ }^{2}$ In addition to the seven previous reports of invasive monitoring during obstetric anaesthesia in PPH referred to by Breen, three additional reports have been published since 1982 in the English language literature. ${ }^{2-4}$ Of the 12 patients included in these reports, eight had vaginal delivery under regional anaesthesia and four were delivered by Caesarean section (three with epidural and one with general anaesthesia). There was one immediate postpartum death at Caesarean section under epidural anaesthesia and seven other deaths occurred, the majority during the first week postpartum. Most of these deaths were sudden and precipitating factors were not obvious. Poor prognostic signs associated with early postpartum death were the presence of antepartum elevation of right atrial pressure with low cardiac output, indicating a significant impairment of right ventricular function. Conversely, survivors all had acceptable cardiac indices and good right ventricular function.

Obstetric indications determine the mode of delivery. Before delivery, a planning conference should be held with all specialties involved in patient management (obstetrics, cardiology, neonatology, critical care, nursing and anaesthesia). If possible, these patients should be delivered at an elected time, and not as an emergency, to allow for coordination of support services.

From published reports, the use of a pulmonary artery

From the Departments of Anaesthesia, McGill University and Royal Victoria Hospital, Montreal, Quebec.

Address correspondence to: Dr. Sally K. Weeks, Department of Anaesthesia, Royal Victoria Hospital, 687 Pine Avenue West, Montreal (Quebec) Canada H3A 1A1. catheter has facilitated intrapartum management of patients with PPH. Unfortunately, this does not seem to be reflected in improved survival. The presence of a pulmonary artery catheter allows for early detection of deterioration in right ventricular function and pharmacological interventions may then be attempted with inotropes and/or pulmonary vasodilators. Some have suggested that a trial of possible therapeutic interventions be done before labour or Caesarean section. ${ }^{5,6}$ There is a characteristically wide variation in individual haemodynamic response to both pharmacological and physiological challenge in PPH. Predelivery assessment of the effect of vasodilators, inotropes, vasopressors, oxytocin and fluid administration may be of value during subsequent anaesthetic management. Pulmonary artery rupture and thrombosis are an increased risk with the use of a pulmonary artery catheter in pulmonary hypertension, but the benefits in such critically ill patients appear to outweigh the disadvantages. 7

It is important to provide excellent analgesia for labour and vaginal delivery, in order to attenuate the cardiovascular effects associated with painful uterine contractions. In theory, a decrease in systemic vascular resistance resulting from epidural local anaesthetic block may result in severe hypotension in a patient with a fixed cardiac output which may be difficult to correct. In practice, epidural anaesthesia has produced good analgesia with minimal problems. The use of intrathecal opioids, perhaps with a continuous catheter technique, would be of particular value in producing excellent analgesia with limited cardiovascular effects. ${ }^{8}$ If the epidural route is chosen, the addition of opioids to an infusion of diluted local anaesthetic appears to offer similar advantages.

Anaesthesia for Caesarean delivery presents an enormous challenge. Epidural anaesthesia has been used with success, but in the presence of pre-existing right ventricular failure, any large decrease in systemic vascular resistance may lead to a further decrease in cardiac output. Refractory hypotension may also cause right ventricular ischaemia, leading to further deterioration in right ventricular function. There is no place for spinal anaesthesia 
for Caesarean section in PPH. The potential hazards of general anaesthesia include increased pulmonary artery pressure during laryngoscopy and intubation, the adverse effects of positive pressure ventilation on venous return and the negative inotropic effects of certain anaesthetic agents. It should be noted that nitrous oxide may further increase pulmonary vascular resistance. However, these adverse effects can be minimized by the use of a narcotic-based induction and maintenance technique. Any resulting narcotic-induced neonatal depression should be easily treated. Intensive postoperative management is of critical importance and should probably continue for one week because of the high incidence of sudden death during this period.

\section{L'anesthésie obstétri- cale en présence d'hy- pertension pulmonaire primaire}

Dans ce numéro, Breen et Janzen rapportent leur succès dans la conduite anesthésique lors d'une césarienne chez une jeune femme atteinte d'hypertension pulmonaire primaire (PPH). Une revue extensive de la pathophysiologie et de la conduite à tenir chez des patients atteints de PPH a récemment été publiée par Rich. ${ }^{1}$ Cette condition très rare peut occasionnellement se présenter lors de la grossesse, particulièrement durant le second et le troisième trimestre, quand la demande hémodynamique devient importante. ${ }^{2}$ En plus des sept rapports cités par Breen sur la surveillance anesthésique invasive lors de l'anesthésie obstétricale dans le PPH, trois rapports additionnels ont été publiés depuis 1982 dans la littérature anglaise. ${ }^{2-4}$ Des douze patientes incluses dans ce rapport, huit ont eu un accouchement naturel sous anesthésie régionale et quatre furent accouchées par césarienne (trois avec épidurale et une sous anesthésie générale). Il y avait un seul décès dans la période postpartum immédiate lors d'une césarienne sous anesthésie épidurale et sept autres décès sont survenus, la majorité durant la première semaine postpartum. La majorité de ces décès furent soudains et des facteurs précipitants n'étaient pas évidents. Parmi les signes de mauvais pronostic en période postpartum précoce, on notait la présence d'augmentation antepartum de la pression de l'oreillette droite avec un bas débit cardiaque, indiquant une diminution significative de la fonction ventriculaire droite. Par contre, les survivantes avaient toutes des indices cardiaques acceptables et une bonne fonction ventriculaire droite.

Les indications obstétricales déterminent le mode d'accouchement. Avant l'accouchement, une conférence planifiée devrait être tenue avec toutes les spécialités impliquées (obstétrique, cardiologie, néonatalogie, intensiviste, soins infirmiers et anesthésie). Si possible, ces patientes doivent être accouchées en période élective et non en urgence, afin de permettre la coordination entre les services de support.

Selon les rapports publiés, l'utilisation d'un cathéter dans l'artère pulmonaire a facilité la conduite durant l'accouchement de ces patientes atteintes de PPH. Malheureusement, ceci ne semble pas se réfléter par une amélioration de la survie. La présence de cathéter dans l'artère pulmonaire permet une détection précoce de la détérioration de la fonction ventriculaire droite et permet des interventions pharmacologiques avec des inotropes et/ou vasodilateurs pulmonaires. Certains ont suggéré d'essayer des interventions thérapeutiques possibles avant la césarienne. ${ }^{5,6}$ Il est caractéristique d'observer de grandes variations individuelles dans la réponse hémodynamique tant à l'intervention pharmacologique que physiologique dans le PPH. L'évaluation avant l'accouchement des effets des vasodilatateurs, inotropes, vasopresseurs, oxytocin et l'administration de liquide peut être de valeur durant la conduite anesthésique subséquente. La rupture de l'artère pulmonaire et la thrombose représentent un risque accru avec l'utilisation du cathéter dans l'artère pulmonaire chez les patients atteints d'hypertension pulmonaire mais les bénéfices chez de tels patients semblent dépasser ces désavantages. ${ }^{7}$

Il est important de fournir une excellente analgésie pour le travail et l'accouchement naturel afin d'atténuer les effets cardiovasculaires associés à la douleur lors des contractions utérines. En théorie, une diminution de la résistance vasculaire systémique avec l'anesthésie épidurale peut amener une hypotension sévère chez ces patientes ayant un débit cardiaque fixe qui pourrait être difficile à corriger. En pratique, l'anesthésie épidurale a produit une bonne analgésie avec des problèmes minimes. L'utilisation d'opiacès par voie intrathécale continue, serait particulièrement intéressante pour l'excellente analgésie et des effets cardiovasculaires limités. ${ }^{8} \mathrm{Si}$ la voie épidurale est choisie, l'addition d'opiacés à la perfusion d'anesthésique local dilué semble offrir des avantages similaires.

L'anesthésie pour césarienne présente un problème énorme. L'anesthésie épidurale a été utilisée avec succès mais en présence d'une défaillance ventriculaire droite pré-existante, toute diminution importante de résistance vasculaire systémique peut amener une plus grande 
diminution du débit cardiaque. L'hypotension réfractaire peut aussi amener une ischémie du ventricule droit, aboutissant à une plus grande détérioration de la fonction ventriculaire droite. Il n'y a pas de place pour l'anesthésie rachidienne pour une césarienne chez les patientes atteintes de PPH. Les dangers potentiels de l'anesthésie générale incluent une augmentation de la pression de l'artère pulmonaire durant la laryngoscopie et l'intubation, les effets néfastes de la ventilation en position positive sur le retour veineux et les effets inotropes négatifs de certains agents anesthésiques. On doit noter que le protoxyde d'azote peut augmenter davantage la résistance du lit vasculaire pulmonaire. Cependant, ces effets néfastes peuvent être minimisés par l'utilisation d'une induction et d'un maintien à base de narcotique. Toute dépression respiratoire néonatale induite par les narcotiques doit être facilement traitée. La conduite postopératoire intensive est d'une importance critique et doit être probablement continuée pour une semaine à cause de la forte incidence de décès soudain durant cette période.

\section{References}

1 Rich S. Primary pulmonary hypertension. Progress in Cardiovascular Diseases 1988; 31: 205-38.

2 Roberts $N$, Keast $P$. Pulmonary hypertension and pregnancy - a lethal combination. Anaesth Intensive Care 1990; 18: 366-74.

3 Takeuchi T, Nishii O, Okamura T et al. Primary pulmonary hypertension in pregnancy. Int J Gynaecol Obstet 1988; 26: 145-50.

4 Feijen $H$, Hein $P$, van Lakwijk-Vondrovicova E et al. Primary pulmonary hypertension and pregnancy. Eur J Obstet Gynecol Reprod Biol 1983; 15: 159-64.

5 Slomka F, Salmeron S, Zetlaoui $P$ et al. Primary pulmonary hypertension and pregnancy: anesthetic management for delivery. Anesthesiology 1988; 69: 959-61.

6 Roessler P, Lambert T. Anaesthesia for Caesarean section in the presence of primary pulmonary hypertension.

Anaesth Intensive Care 1986; 14: 317-20.

7 Barash P, Nardi D, Hammond G et al. Catheter-induced pulmonary artery perforation. J Thorac Cardiovasc Surg 1981; 82: 5-12.

8 Abboud T, Raya J, Nouehied $R$ et al. Intrathecal morphine for relief of labor pain in a parturient with severe pulmonary hypertension. Anesthesiology 1983; 59: $477-9$. 